\title{
Correction to: Bilingualism Is Associated with a Delayed Onset of Dementia but Not with a Lower Risk of Developing it: a Systematic Review with Meta-Analyses
}

\author{
Stefano Brini ${ }^{1,2,3,10}$ (D) Hamid R. Sohrabi ${ }^{1,4,5} \cdot$ Jeffrey J. Hebert ${ }^{1,6} \cdot$ Mitchell R. L. Forrest $^{1} \cdot$ Matti Laine $^{2,7} \cdot$ \\ Heikki Hämäläinen ${ }^{2,10} \cdot$ Mira Karrasch $^{7} \cdot$ Jeremiah J. Peiffer $^{1} \cdot$ Ralph N. Martins ${ }^{4,5,8} \cdot$ Timothy J. Fairchild $^{1,9}$
}

Published online: 13 March 2020

(C) The Author(s) 2020

\section{Correction to: Neuropsychology Review}

https://doi.org/10.1007/s11065-020-09426-8

The original version of this article unfortunately contained the following mistakes.

1. In the Results section under the paragraph Disease Severity, the sentence "The PIs ranged between -0.47 and 0.57 MMSE points" should read -0.49 and 0.59 MMSE points.

2. In Figs. 3, 5, and 7, the labels "favour bilinguals" and "favours monolinguals" should be inverted. Therefore, it should be "favours monolinguals" and "favours bilinguals". Please see below for the correct figures.

The online version of the original article can be found at https://doi.org/ 10.1007/s11065-020-09426-8

Stefano Brini

stefano.brini@city.ac.uk

1 Discipline of Psychology, Exercise Science, Chiropractic and Counselling, Murdoch University, Perth, Western Australia, Australia

2 Turku Brain and Mind Center, Turku, Finland

3 Health Services Research and Management School of Health Sciences, City, University of London, London, UK

4 School of Medical and Health Sciences, Edith Cowan University, Perth, Western Australia, Australia
Department of Biomedical Sciences, Macquarie University, Macquarie Park, New South Wales, Australia

6 Faculty of Kinesiology, University of New Brunswick, Fredericton, Canada

7 Department of Psychology, Åbo Akademi University, Turku, Finland

8 Australian Alzheimer's Research Foundation, Perth, Western Australia, Australia

9 Centre for Molecular Medicine and Innovative Therapeutics, Murdoch University, Perth, Western Australia, Australia

10 Department of Psychology and Speech-Language Pathology, University of Turku, Turku, Finland 


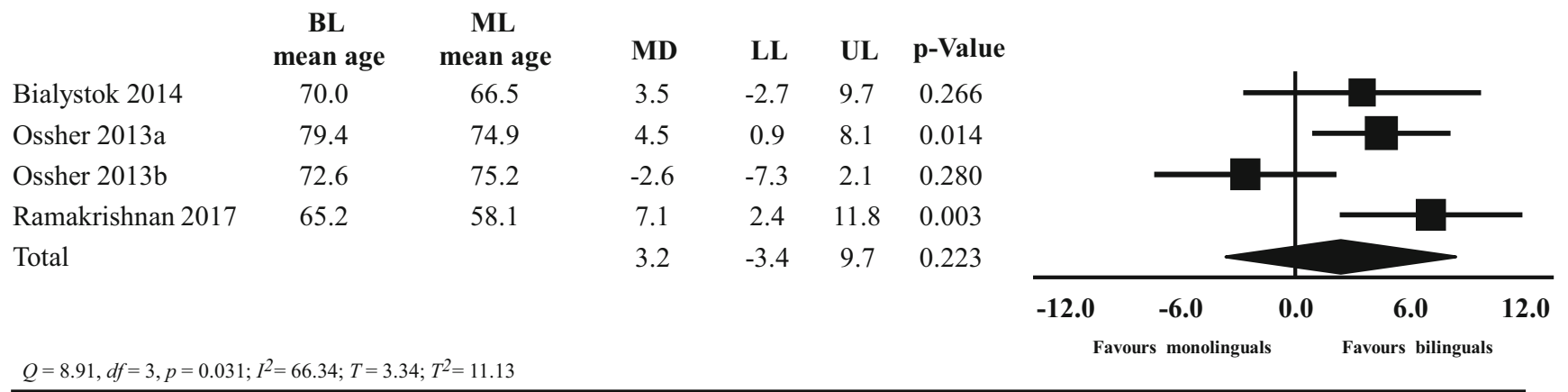

Fig. 3 Forest plot showing the mean difference (MD) in the age of MCI diagnosis between bilinguals (BL) and monolinguals (ML); MCI: Mild cognitive impairment; LL: lower limit, UP: upper limit; CI: confidence interval

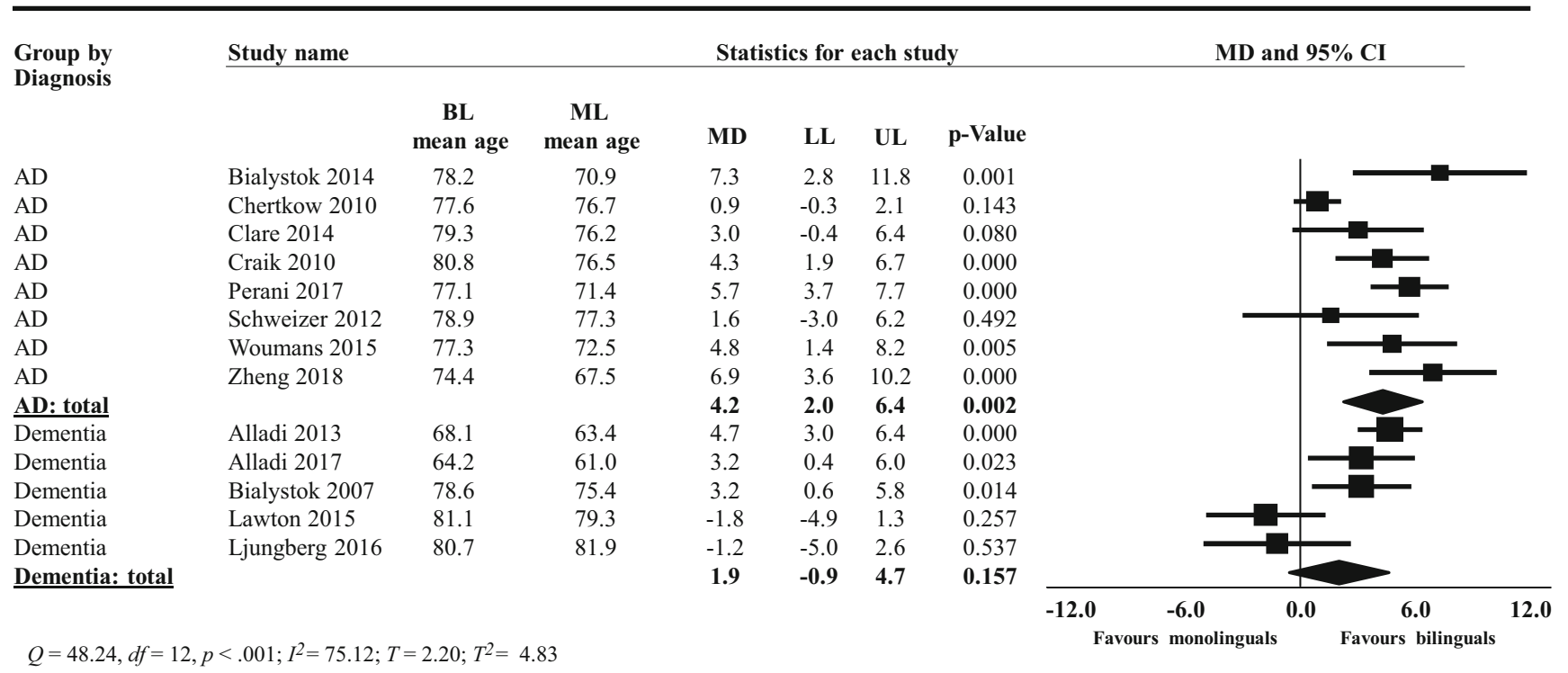

Fig. 5 Forest plot showing the mean difference (MD) in the subgroup meta-analysis comparing studies including participants with AD to studies including participants with dementia on the age of $\mathrm{AD}$ and dementia diagnosis between bilinguals (BL) and monolinguals (ML); AD: Alzheimer's disease; LL: lower limit, UP: upper limit; CI: confidence interval 


Study name Statistics for each study Hedges's g and 95\% CI

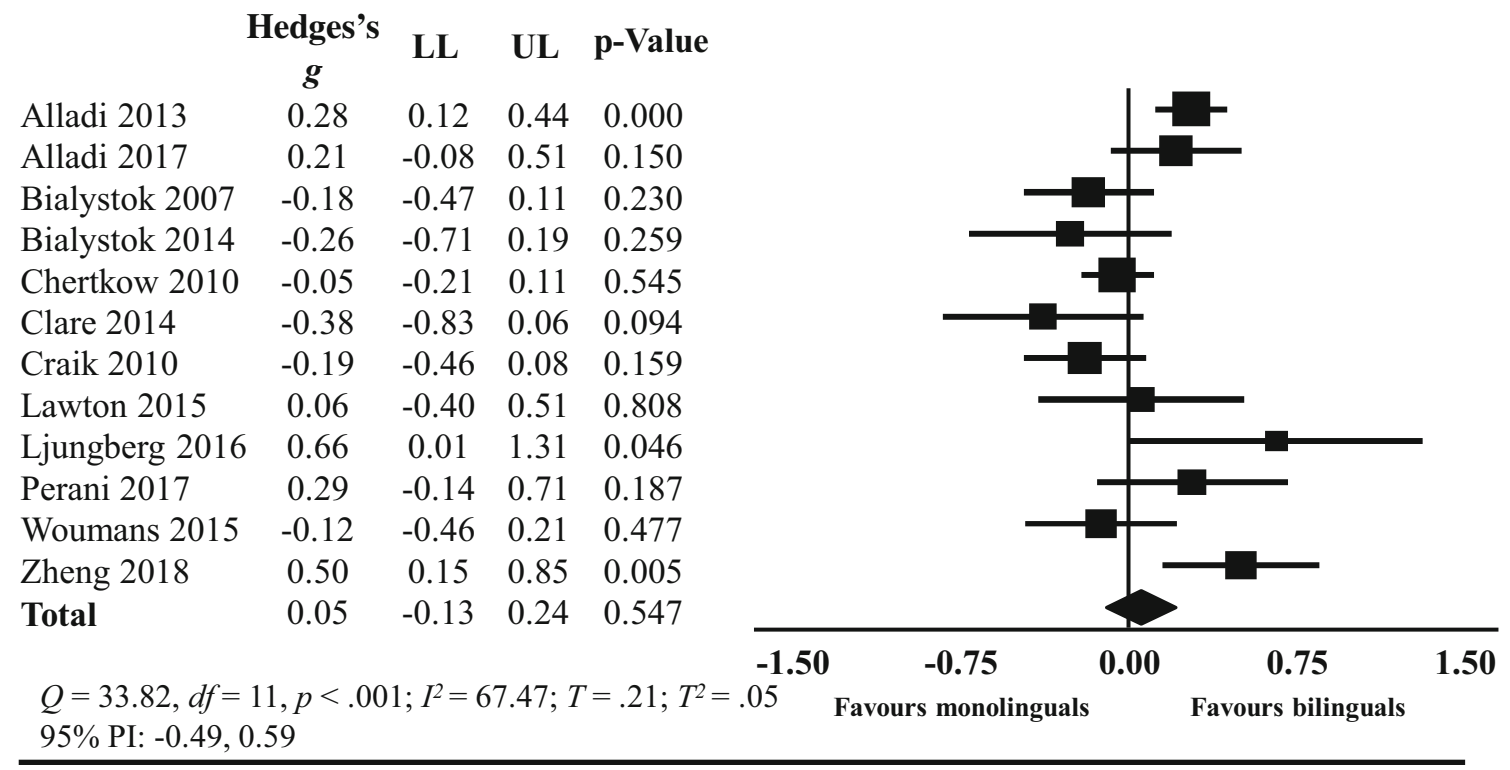

Fig. 7 Forest plot showing the standardized mean difference (Hedges's $g$ ) in the degree of disease severity at dementia diagnosis between bilinguals (BL) and monolinguals (ML); LL: lower limit, UP: upper limit; CI: confidence interval

Open Access This article is licensed under a Creative Commons Attribution 4.0 International License, which permits use, sharing, adaptation, distribution and reproduction in any medium or format, as long as you give appropriate credit to the original author(s) and the source, provide a link to the Creative Commons licence, and indicate if changes were made. The images or other third party material in this article are included in the article's Creative Commons licence, unless indicated otherwise in a credit line to the material. If material is not included in the article's Creative Commons licence and your intended use is not permitted by statutory regulation or exceeds the permitted use, you will need to obtain permission directly from the copyright holder. To view a copy of this licence, visit http://creativecommons.org/licenses/by/4.0/. 